\title{
Determinants of stunting in Indonesian children: evidence from a cross-sectional survey indicate a prominent role for the water, sanitation and hygiene sector in stunting reduction
}

\author{
Harriet Torlesse ${ }^{1 *}$, Aidan Anthony Cronin', Susy Katikana Sebayang ${ }^{2}$ and Robin Nandy ${ }^{1}$
}

\begin{abstract}
Background: Stunting in early life has considerable human and economic costs. The purpose of the study was to identify factors associated with stunting among children aged 0-23 months in Indonesia to inform the design of appropriate policy and programme responses.

Methods: Determinants of child stunting, including severe stunting, were examined in three districts in Indonesia using data from a cross-sectional survey conducted in 2011. A total of 1366 children were included. The analysis used multiple logistic regression to determine unadjusted and adjusted odds ratios.

Results: The prevalence of stunting and severe stunting was $28.4 \%$ and $6.7 \%$, respectively. The multivariate analysis on determinants of stunting identified a significant interaction between household sanitary facility and household water treatment ( $P$ for interaction $=0.007$ ) after controlling for potential covariates: in households that drank untreated water, the adjusted odds on child stunting was over three times higher if the household used a unimproved latrine (adjusted odds ratio 3.47, $95 \%$ confidence interval 1.73-7.28, $P<0.001$ ); however, in households that drank treated water, the adjusted odds on child stunting was not significantly higher if the household used an unimproved latrine (adjusted odds ratio 1.27, $95 \%$ confidence interval 0.99-1.63, $P=0.06$ ). Other significant risk factors included male sex, older child age and lower wealth quintile. The risk factors for severe stunting included male sex, older child age, lower wealth quintile, no antenatal care in a health facility, and mother's participation in decisions on what food was cooked in the household.

Conclusions: The combination of unimproved latrines and untreated drinking water was associated with an increased odds on stunting in Indonesia compared with improved conditions. Policies and programmes to address child stunting in Indonesia must consider water, sanitation and hygiene interventions. Operational research is needed to determine how best to converge and integrate water, sanitation and hygiene interventions into a broader multisectoral approach to reduce stunting in Indonesia.
\end{abstract}

Keywords: Indonesia, Stunting, Sanitation, Household water treatment

\footnotetext{
* Correspondence: htorlesse@unicef.org

'UNICEF Indonesia, World Trade Center 6 (10th Floor), Jalan Jenderal

Sudirman Kav. 31, Jakarta 12920, Indonesia

Full list of author information is available at the end of the article
} 


\section{Background}

Failure to grow and develop optimally in early life has considerable human and economic costs [1]. Stunting increases the risk of child deaths, adversely affects cognitive and motor development, lowers performance at school, increases the risk of overnutrition and noncommunicable diseases, and reduces productivity in adulthood [2]. These accumulative effects cost African and Asian countries up to $11 \%$ of their gross national product [3].

Stunting, or low height for age, is caused by long-term insufficient nutrient intake and/or frequent infections. Despite Indonesia's middle income status, the country carries the fifth highest burden of stunted children in the world [4]. There has been negligible change in the stunting prevalence in the last decade, and if current trends continue, the country is unlikely to achieve the 2012 World Health Assembly goal to reduce stunting by $40 \%$ by 2025 [1]. Over one-third (37\%) of children aged less than five years were stunted in 2013 and the prevalence exceeded $40 \%$ in 15 out of 33 provinces; $18 \%$ of children were severely stunted [5].

Indonesia joined the global Scaling Up Nutrition (SUN) Movement in 2011 and launched its national movement in 2013 to galvanize action across multiple sectors to reduce stunting and other forms of undernutrition. Both the widely accepted conceptual framework for optimal nutrition [2] and the policy framework for the SUN Movement in Indonesia [6] recognize the need for a multisector actions. However, information on the determinants on stunting in Indonesia to inform the design of multi-sector programmes is lacking, and the operationalization of multisector response is yet to be realised [7].
There is close symmetry between the provincial-level maps for sanitation and child stunting in Indonesia (Fig. 1). Using data collected by the 2013 Basic Health Research Survey [5], we determined that provincial estimates of the proportion of households with access to an improved latrine are inversely correlated with provincial estimates of the percentage of stunted children aged less than five years $\left(R^{2}=65.7 \%, P<0.001\right)$. To examine the relationship further, this study utilized data from a cross-sectional survey in three districts in Indonesia to examine maternal, child and household determinants of stunting and severe stunting in children, including indicators on household water, sanitary and hygiene (WASH) facilities and practices.

\section{Methods}

Subjects

The analysis used data from a baseline survey conducted in three districts in Indonesia for the European Union funded Maternal and Young Child Nutrition Security Initiative (MYCNSIA) project between November and December 2011. The MYCNSIA project was designed to reduce stunting in children aged less than three years and anaemia in pregnant women through the scale-up of nutrition-specific and nutrition-sensitive interventions.

The three MYCNSIA focus districts were selected to represent three different typologies in Indonesia: Sikka is a coastal district in East Nusa Tenggara Province that has one of the highest stunting prevalence figures in the country; Jayawijaya is a remote highland district in Papua Province where many social and health indictors are well below the national average; and Klaten is a densely populated district in Central Java where the burden of stunting is high.
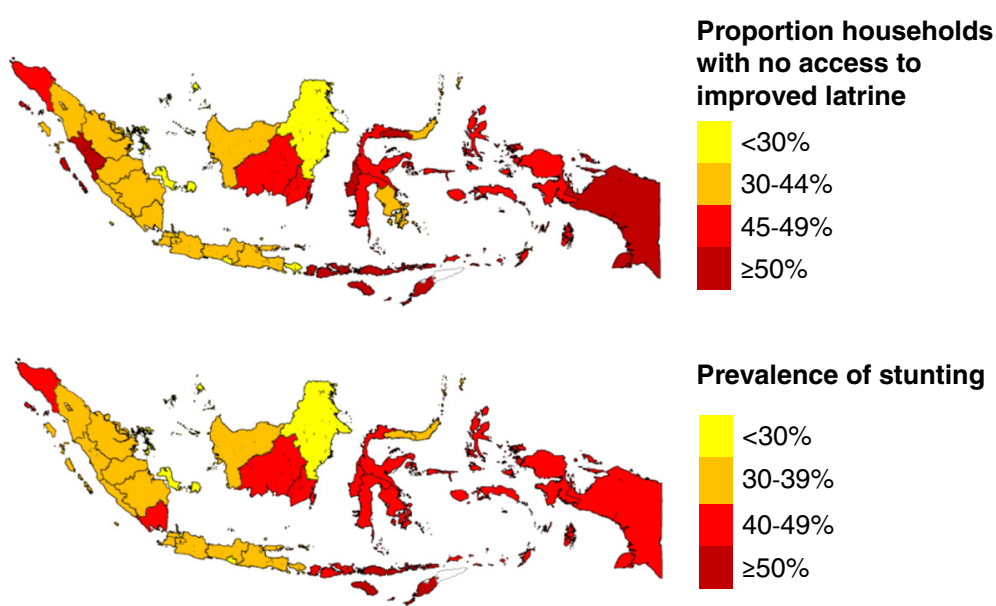

Prevalence of stunting

$<30 \%$

$30-39 \%$

$40-49 \%$

$\geq 50 \%$

Fig. 1 Proportion of households without access to an improved latrine and prevalence of stunting in child stunting. Provincial estimates of the proportion of households without access to an improved latrine and the prevalence of stunting in children aged 0-59 months in Indonesia (derived from [5]) 
Households were eligible for inclusion in the survey if they contained a child aged 0-35 months and if the primary caregiver was present for interview. Children were excluded if a household member reported that they had a chronic disease lasting more than three months such as cerebral palsy, asthma or diabetes.

\section{Sampling design}

The survey used a multistage cluster sampling design to produce statistically representative data for the three districts as a single sample universe. A desired sample size of 2009 children aged 0-35 months was calculated to detect a 5 percentage point decline in the estimated baseline stunting prevalence of $46.4 \%$ using a one-tail test at a power of $80 \%$, confidence level of $95 \%$, design effect of 1.5 and with $10 \%$ additional children to allow for missing data. A total of 102 clusters, comprising census blocks created by the Indonesia Central Bureau of Statistics, were selected from the three districts using probability proportionate to size sampling (70 clusters in Klaten, 19 in Sikka and 13 in Jayawijaya). In each cluster, 20 households were selected using simple random sampling from a list of households with children aged 0-35 months in each census block. If the cluster did not have 20 eligible households, a contiguous cluster was visited to randomly select the missing number of households; the final number of census blocks was 193 (148 in Klaten, 25 in Sikka and 20 in Jayawijaya). If a household had more than one child aged 0-35 months, only the youngest child was included.

\section{Data collection}

A structured pre-tested questionnaire was used to record data on children aged 0-35 months, their mothers, and their households. The data included the child's age, sex, weight and height/length; mother's age, level of education and participation in household decisions; breastfeeding, complementary feeding and handwashing practices; child's access to health and nutrition services (growth monitoring, micronutrient supplementation, and immunization); mother's access to health services (antenatal care, assistance at delivery and place of delivery); household water, sanitation and hygiene; and socio-economic characteristics of the household.

Child weight was measured using SECA electronic scales to a precision of $0.1 \mathrm{~kg}$ and child length (for children aged 0-23 months) or height (for children aged 24-35 months) was measured using a locally made height/length board to a precision of $0.1 \mathrm{~cm}$. The SECA weighing scales were calibrated every morning, prior to data collection, using a $5 \mathrm{~kg}$ standard weight. Duplicate measurements of anthropometry were performed for $10 \%$ of samples; the within-subject coefficient of variation from the duplicate measurements in both children and women was less than $5 \%$. All enumerators received at least two days training prior to data collection, and those responsible for taking anthropometric measurements received an additional day of training. Supervisors were assigned to oversee the work of the enumerators and facilitate good rapport with community members.

\section{Data entry and analysis}

Data entry operators entered the data using EPI Info version 7.0.8.0 (Centre for Disease Control, Atlanta, Georgia, USA). Approximately $10 \%$ of the questionnaires were entered in duplicate and keystroke errors were found in less than $0.7 \%$ of fields. A series of checks were performed for duplicate entries, outliers and missing data.

Data analysis was conducted using Stata 11.0 (StataCorp, College Station, Texas, USA). Data were adjusted for cluster sampling using the Generalized Estimating Equation model [8] and robust estimates with xtgee command in Stata 11.0. The analysis focused on children aged 0-23 months because data on infant and young child feeding (IYCF) practices and access of mother to antenatal and delivery care services were not available for children aged 24-35 months and these indicators were potential determinants of stunting. The analysis was repeated for children aged 0-35 months with the caveat that indicators on IYCF practices and maternal health care were not included. Children with missing or invalid data for any of the variables examined were not included in the analytical sample.

Stunting and severe stunting were defined as the proportion of children whose weight-for-height $\mathrm{z}$-score was below -2 standard deviations and -3 standard deviations, respectively, of the median height-for-age of the World Health Organization (WHO) Child Growth Standards [9].

WHO definitions for indicators of IYCF practices in children aged less than two years were applied [10]. In addition, we constructed a variable on "age-appropriate feeding" that combined exclusive breastfeeding for children aged 0-5 months with minimum acceptable diet for children aged 6-23 month; a child was considered to have age-appropriate feeding if she/he was aged 0-5 months and exclusively breastfed or aged 6-23 months and consumed a minimal acceptable diet.

An index of household wealth was constructed using data on household assets (materials used to construct the floor, walls and roof of the house, fuel for cooking, water source, sanitation facility, and ownership of livestock, vehicles and electronic goods) and five quintile categories were created.

The operational definitions used by the UNICEF and WHO's Joint Monitoring Programme for improved drinking water supply, treated water, and improved sanitation 
facilities were applied [11]. The household water source is considered "improved" if it was piped into the dwelling, yard or plot, a public tap, a protected well in the dwelling, yard or plot, a protected public well, or rainwater. The household water is considered "treated" if the household reported that it was boiled, bleached, filtered or solar disinfected. The household sanitary facility is considered "improved" only if it was a latrine with a septic tank. The disposal of a child's faeces was considered safe only if it was discarded in an improved latrine.

In models using stunting or severe stunting as the dependent variables, we report on unadjusted odds ratios (OR) and adjusted odds ratios (AOR) and $95 \%$ confidence intervals (CI) for variables. The selection of variables was based on the conceptual framework for optimal nutrition [2]. Multiple logistic regression included variables that had a $P$-value $<0.25$ in the univariate analysis. Variables were analysed using the backward elimination and those with a $P$-value $<0.05$ in multivariate analysis were retained in the final logistic regression model. For all tests, a $P$-value $<0.05$ was considered statistically significant. Interactions were considered significant for $P$ values $<0.01$.

\section{Results}

The survey included a representative sample of 2023 children aged 0-35 months, including 1424 children aged 0-23 months. Unless otherwise stated, the analysis presented here relates to 1366 (95.9\%) children aged 0-23 months whose mother was available for interview and who had valid data for all variables examined. Valid stunting data was missing for 3 children. Information on the characteristics of the mother, child or household were unavailable for a further 55 children, either because the mother was not available at the time of interview or the mother was unable to provide this information. Of these 55 children, 13 (23.6\%) were stunted.

\section{Characteristics of the sample}

Table 1 provides data on the socio-economic characteristics of the mothers of children aged 0-23 months and their households. Four percent of mothers were aged less than 20 years, and $14.4 \%$ of mothers did not complete primary education. Less than two-thirds of households $(61.1 \%)$ had access to an improved latrine, and $43.4 \%$ practiced safe disposal of children's faeces. Only $55.3 \%$ of households reported that they used soap for handwashing. Less than one third (31.6\%) of households had an improved source of drinking water, however, $90.0 \%$ of households reported that they treated their water before drinking. Coverage of antenatal care (ANC) by mothers of the children during their most recent pregnancy was high: around $95 \%$ of mothers accessed ANC at least four times during pregnancy,
Table 1 Socio-economic characteristics of the population $(N=1366)$

\begin{tabular}{|c|c|}
\hline & Proportion (\%) \\
\hline \multicolumn{2}{|l|}{ Mother's age } \\
\hline$<20$ years & 4.0 \\
\hline 20-29 years & 51.5 \\
\hline 30-39 years & 37.8 \\
\hline$\geq 40$ years & 6.7 \\
\hline \multicolumn{2}{|l|}{ Mothers' education } \\
\hline No or incomplete primary education & 14.4 \\
\hline Completed primary education & 16.8 \\
\hline Completed junior high education & 24.4 \\
\hline Completed senior high education & 44.5 \\
\hline \multicolumn{2}{|l|}{ Number of household members } \\
\hline$\leq 4$ people & 40.3 \\
\hline$>4$ people & 59.7 \\
\hline \multicolumn{2}{|l|}{ Household water, sanitation and hygiene } \\
\hline Improved sanitary facility & 61.1 \\
\hline Safe disposal of child's faeces & 43.4 \\
\hline Use of soap for handwashing & 55.3 \\
\hline Improved source of drinking water & 31.6 \\
\hline Treated water & 90.0 \\
\hline \multicolumn{2}{|l|}{ Mother's care during last pregnancy and delivery } \\
\hline At least 4 antenatal care visits & 93.3 \\
\hline Antenatal care provided by doctor/midwife & 95.3 \\
\hline Antenatal care in a private or public health facility & 96.5 \\
\hline \multicolumn{2}{|l|}{ Mother participates in household decisions } \\
\hline Household purchases on food & 88.7 \\
\hline What food to cook for the household & 89.5 \\
\hline What food to give to child & 95.2 \\
\hline Seeking health care for child & 86.4 \\
\hline \multicolumn{2}{|l|}{ Wealth quintile } \\
\hline Lowest & 21.5 \\
\hline Second & 20.3 \\
\hline Third & 20.0 \\
\hline Fourth & 18.7 \\
\hline Highest & 19.5 \\
\hline
\end{tabular}

received ANC from a doctor or midwife, and in a private or public health facility. Mothers' participation in household decisions was also high: $88.7 \%$ mothers participated in household decisions on food, $89.5 \%$ on what food to cook for the household; $95.2 \%$ on what food was given to the child; and $86.4 \%$ on seeking health care for the child.

Table 2 provides data on the nutritional status of the children and infant and young child feeding practices. The prevalence of total stunting (moderate and severe) 
Table 2 Background characteristics of children aged 0-23 months ( $N=1366$ unless otherwise stated)

\begin{tabular}{|c|c|}
\hline & Proportion (\%) \\
\hline \multicolumn{2}{|l|}{ Sex } \\
\hline Girl & 49.6 \\
\hline Boy & 50.4 \\
\hline \multicolumn{2}{|l|}{ Age } \\
\hline $0-5$ months & 22.6 \\
\hline 6-11 months & 25.0 \\
\hline $12-23$ months & 52.4 \\
\hline \multicolumn{2}{|l|}{ Stunting } \\
\hline Moderate stunting & 21.7 \\
\hline Severe stunting & 6.7 \\
\hline Total stunting & 28.4 \\
\hline \multicolumn{2}{|l|}{ IYCF practices } \\
\hline $\begin{array}{l}\text { Breastfeeding initiated within one hour of birth } \\
\text { (children 0-23 months) }\end{array}$ & 67.9 \\
\hline Exclusive breastfeeding (children 0-5 months) ${ }^{a}$ & 54.1 \\
\hline $\begin{array}{l}\text { Minimum dietary diversity of complementary food } \\
\text { (children 6-23 months) }^{b}\end{array}$ & 48.3 \\
\hline $\begin{array}{l}\text { Minimum dietary frequency of complementary food } \\
\text { (children 6-23 months) }^{\mathrm{b}}\end{array}$ & 73.6 \\
\hline Minimum acceptable diet (children 6-23 months) ${ }^{b}$ & 36.6 \\
\hline Age-appropriate feeding (children 0-23 months) & 40.6 \\
\hline
\end{tabular}

${ }^{\mathrm{a}} N=309 ;{ }^{\mathrm{b}} N=1057$

was $28.4 \%$ and the prevalence of severe stunting was $6.7 \%$. About two-thirds $(67.9 \%)$ of children were breastfed within one hour of birth, and $54.1 \%$ of children aged less than six months were exclusively breastfed. Only $36.6 \%$ of children aged 6-23 months were fed a minimum acceptable diet comprising adequate number of milk feeds, meals and food groups. Overall, $40.6 \%$ of children aged 0-23 months had age-appropriate feeding, defined here as exclusive breastfeeding for children aged 0-5 months and minimum acceptable diet for children aged 6-23 months.

\section{Risk factors for stunting}

Table 3 provides the results of the bivariate and multivariate analysis on the association of total stunting with child, maternal and household characteristics.

Using bivariate analysis, the prevalence of stunting was significantly higher among children aged 12-23 months $(37.7 \%)$ and children aged 6-11 months (22.6\%) than children aged 0-5 months (13.3\%). Children who were not given age-appropriate feeding were significantly more likely to be stunted than those who were fed appropriately (31.3\% vs. $24.2 \%$ ). The prevalence of stunting was higher among children whose mothers had not completed primary education (43.4\%) or completed primary education $(31.0 \%)$ compared with those who had completed high school (23.0\%). Children of poorer households, as measured by an asset-based wealth quintile, were significantly more likely to be stunted than wealthier households; the prevalence of stunting ranged from $19.2 \%$ among the highest quintile to $40.1 \%$ among the lowest wealth quintile. The stunting prevalence was significantly higher among children living in households that had an unimproved latrine compared with an improved latrine (35.3\% vs. $24.0 \%$ ); households that did not use soap for handwashing compared with those that did (31.6\% vs. $25.8 \%$ ); and households that drank untreated water compared with treated (38.2 \% vs. $27.3 \%)$. The stunting prevalence was also significantly lower among children of mothers who had good access to health care as indicated by an inadequate number of ANC visits, ANC care by a doctor or midwife, and ANC at a health facility.

Three variables were independently significant when entered into the multiple logistic model to control for potential confounding: child sex, child age, and household wealth quintile. Boys had a $45 \%$ higher odds on being stunted than girls (AOR 1.45; $95 \%$ CI 1.111.90). Children 12-23 months had an over four-fold odds on stunting than children aged 0-5 months (AOR 4.40; $95 \%$ 2.97-6.53). Children from the lowest wealth quintile had more than twice the odds on being stunted than children from the highest wealth quintile (AOR 2.30; 95 \% CI 1.43-3.68). In addition, there was an interaction between household sanitary facility and water treatment ( $P$ for interaction $<0.007$ ): among children who lived in households that drank untreated water, the adjusted odds on stunting was over three times greater if the household used a unimproved latrine (AOR 3.47, $95 \%$ CI 1.73-7.28, P<0.001); however, among children who lived in households that drank treated water, the adjusted odds on stunting was not significantly higher if the household used an unimproved latrine (AOR 1.27, $95 \%$ CI 0.99-1.63, $P=0.06$ ).

We repeated the multivariate analysis for the dataset of children aged 0-35 months $(N=1937)$, excluding variables that were not available for children aged 24-35 months (IYCF practices and mother's access to ANC during her most recent pregnancy). The same three variables were significant in the multiple logistic regression model (child sex, child age, wealth quintile) as well as the interaction between household sanitation facility and household water treatment (see Additional file 1: Table S1). Adjusting for other significant covariates, unimproved sanitation in household with untreated water was associated with more than twice the odds of stunting in their children (AOR 2.60, $95 \%$ CI 1.37-4.93, $P=$ $0.004)$, while unimproved sanitation in households that treated their water was associated with only $28 \%$ more odds of stunting (AOR 1.28, $95 \%$ CI 1.04-1.58, $P=$ 
Table 3 Risk factors for total stunting in children aged 0-23 months

\begin{tabular}{|c|c|c|c|c|c|c|c|c|c|}
\hline \multirow[t]{2}{*}{ Factors } & & \multirow{2}{*}{$\begin{array}{l}\text { Stunted } \\
(\%)\end{array}$} & \multirow[t]{2}{*}{ N } & \multicolumn{3}{|c|}{ Unadjusted (bivariate) } & \multicolumn{3}{|c|}{ Adjusted (multivariate) } \\
\hline & & & & $\overline{O R}$ & $(95 \% \mathrm{Cl})$ & $P$ & $\overline{\mathrm{OR}}$ & $(95 \% \mathrm{Cl})$ & $P$ \\
\hline \multirow[t]{2}{*}{ Sex } & Boys & 30.9 & 689 & 1.29 & $(0.99-1.68)$ & 0.06 & 1.45 & $(1.11-1.90)$ & 0.007 \\
\hline & Girls & 25.8 & 677 & 1.00 & & & 1.00 & & \\
\hline \multirow[t]{3}{*}{ Age of child } & $12-23$ months & 37.7 & 716 & 4.00 & $(2.72-5.87)$ & $<0.001$ & 4.40 & $(2.97-6.53)$ & $<0.001$ \\
\hline & 6-11 months & 22.6 & 341 & 1.91 & $(1.27-2.88)$ & & 1.97 & $(1.30-2.99)$ & \\
\hline & $0-5$ months & 13.3 & 309 & 1.00 & & & 1.00 & & \\
\hline \multirow[t]{2}{*}{ Breastfeeding within one hour of birth } & No & 30.1 & 438 & 1.14 & $(0.87-1.50)$ & 0.34 & & & \\
\hline & Yes & 27.6 & 928 & 1.00 & & & & & \\
\hline \multirow[t]{2}{*}{ Age-appropriate feeding } & No & 31.3 & 812 & 1.39 & $(1.09-1.77)$ & 0.008 & & & \\
\hline & Yes & 24.2 & 554 & 1.00 & & & & & \\
\hline \multirow[t]{4}{*}{ Mother's age } & $\geq 40$ years & 28.3 & 92 & 1.26 & $(0.61-2.63)$ & 0.79 & & & \\
\hline & 30-39 years & 29.3 & 516 & 1.38 & $(0.73-2.60)$ & & & & \\
\hline & 20-29 years & 28.1 & 704 & 1.29 & $(0.70-2.37)$ & & & & \\
\hline & $<20$ years & 24.1 & 54 & & & & & & \\
\hline \multirow[t]{4}{*}{ Mother's education } & No or incomplete primary & 43.4 & 196 & 2.53 & $(1.77-3.62)$ & $<0.001$ & & & \\
\hline & Completed primary & 31.0 & 229 & 1.48 & $(1.04-2.12)$ & & & & \\
\hline & Completed junior high & 27.6 & 333 & 1.28 & $(0.96-1.72)$ & & & & \\
\hline & Completed senior high & 23.0 & 608 & 1.00 & & & & & \\
\hline \multirow[t]{2}{*}{ Number of household members } & $>4$ & 28.9 & 816 & 1.03 & $(0.83-1.29)$ & 0.77 & & & \\
\hline & $\leq 4$ & 27.6 & 550 & 1.00 & & & & & \\
\hline \multirow[t]{5}{*}{ Wealth quintile } & Lowest & 40.1 & 294 & 2.84 & $(1.84-4.40)$ & $<0.001$ & 2.30 & $(1.43-3.68)$ & 0.004 \\
\hline & Second & 31.0 & 277 & 1.89 & $(1.33-2.70)$ & & 1.85 & $(1.26-2.72)$ & \\
\hline & Third & 27.1 & 273 & 1.56 & $(1.06-2.30)$ & & 1.68 & $(1.11-2.55)$ & \\
\hline & Fourth & 23.0 & 256 & 1.26 & $(0.83-1.91)$ & & 1.31 & $(0.85-2.04)$ & \\
\hline & Highest & 19.2 & 266 & 1.00 & & & 1.00 & & \\
\hline \multirow[t]{2}{*}{ Sanitation } & Unimproved & 35.3 & 532 & 1.71 & $(1.37-2.15)$ & $<0.001$ & 1.27 & $(0.99-1.63)$ & 0.06 \\
\hline & Improved & 24.0 & 834 & 1.00 & & & 1.00 & & \\
\hline \multirow[t]{2}{*}{ Safe disposal of child's faeces } & Unsafe & 29.6 & 773 & 1.10 & $(0.87-1.40)$ & 0.41 & & & \\
\hline & Safe & 26.8 & 593 & 1.00 & & & & & \\
\hline \multirow[t]{2}{*}{ Use of soap for hand washing } & Not use soap & 31.6 & 611 & 1.29 & $(1.00-1.67)$ & 0.05 & & & \\
\hline & Use soap & 25.8 & 755 & 1.00 & & & & & \\
\hline \multirow[t]{2}{*}{ Water source } & Unimproved & 27.3 & 935 & 0.86 & $(0.67-1.10)$ & 0.23 & & & \\
\hline & Improved & 30.9 & 431 & 1.00 & & & & & \\
\hline \multirow[t]{2}{*}{ Water treatment } & Untreated & 38.2 & 136 & 1.59 & $(1.08-2.34)$ & 0.019 & 0.89 & $(0.49-1.61)$ & 0.70 \\
\hline & Treated & 27.3 & 1230 & 1.00 & & & 1.00 & & \\
\hline \multirow{2}{*}{$\begin{array}{l}\text { Number of ANC visits of mother during } \\
\text { pregnancy }\end{array}$} & $<4$ & 40.7 & 91 & 1.70 & $(1.12-2.60)$ & 0.013 & & & \\
\hline & $\geq 4$ & 27.5 & 1275 & 1.00 & & & & & \\
\hline \multirow{2}{*}{$\begin{array}{l}\text { Doctor/midwife provided ANC to mother } \\
\text { during pregnancy }\end{array}$} & No & 45.3 & 64 & 2.07 & $(1.29-3.33)$ & 0.003 & & & \\
\hline & Yes & 27.6 & 1302 & 1.00 & & & & & \\
\hline \multirow[t]{2}{*}{ ANC in private or public health facility } & No & 45.8 & 48 & 2.12 & $(1.16-3.87)$ & 0.014 & & & \\
\hline & Yes & 27.8 & 1318 & 1.00 & & & & & \\
\hline \multirow{2}{*}{$\begin{array}{l}\text { Mother participates in decisions on } \\
\mathrm{HH} \text { food purchases }\end{array}$} & Yes & 28.8 & 1211 & 1.21 & $(0.78-1.87)$ & 0.39 & & & \\
\hline & No & 25.2 & 155 & 1.00 & & & & & \\
\hline
\end{tabular}


Table 3 Risk factors for total stunting in children aged 0-23 months (Continued)

\begin{tabular}{llllllll}
\hline Mother participates in decisions on what & Yes & 29.2 & 1223 & 1.50 & $(0.95-2.38)$ & 0.08 \\
food is cooked for HH & No & 21.7 & 143 & 1.00 & & & \\
Mother participates in decisions on food & Yes & 28.5 & 1300 & 1.18 & $(0.61-2.27)$ & 0.63 \\
given to child & No & 25.8 & 66 & 1.00 & & & \\
Mother participates in decisions on seeking & Yes & 28.7 & 1180 & 1.13 & $(0.79-1.61)$ & 0.50 \\
Mealth care for child & No & 26.3 & 186 & 1.00 & & $2.81 \quad(1.32-5.97)$ & 0.007 \\
\hline
\end{tabular}

0.02). In addition, the odds on being stunted were significantly greater among children whose mothers did not complete primary education compared with those who completed senior high school (AOR 1.67, $95 \% \mathrm{CI}$ $1.13-2.47)$.

\section{Risk factors for severe stunting}

Table 4 provides the results for severe stunting children aged 0-23 months. Using bivariate analysis, the prevalence of severe stunting was significantly higher among children aged 12-23 months (8.8\%) than children aged 0-5 months (2.9\%). The prevalence of severe stunting was higher among children whose mothers had not completed primary education (19.4\%) or completed primary education (8.3\%) compared with those who completed high school (3.0\%). Children of poorer households were significantly more likely to be severely stunted than wealthier households; the prevalence ranged from $2.3 \%$ among the highest quintile to $17.0 \%$ among the lowest wealth quintile. The severe stunting prevalence was significantly higher among children living in households that had unimproved latrines compared with improved (10.5\% vs. $4.3 \%$ ); households that did not safely dispose of child faeces compared to those that did (8.9\% vs. $3.9 \%)$; households that did not use soap for handwashing compared with those that did (10.0\% vs. $4.1 \%$ ); and households that drank untreated water compared with treated (14.7\% vs. $5.9 \%)$. The severe stunting prevalence was significantly lower among children of mothers who had good access to health care as indicated by ANC care by a doctor or midwife and at a health facility. Children whose mothers participated in decisions on what food was cooked for the household had a higher prevalence of severe stunting than children of mothers who did not participate in these decisions (7.3 \% vs. $2.1 \%$ ).

Four variables were independently significant when entered into the multiple logistic model to control for potential confounding: child sex, child age, mother's education, and ANC care in a health facility. Mother's education and wealth quintile were strongly associated (Pearson $X^{2}=597, P<0.001$ ); when mother's education was excluded from the multivariate analysis, wealth quintile was significantly associated with severe stunting and there was little change in the AOR of other variables. Given that it is easier to reverse low wealth status than poor education status, we retained wealth quintile in the multivariate analysis (Table 4). Boys had a $77 \%$ higher odds on being severely stunted than girls (AOR 1.77; $95 \%$ CI 1.07-2.93). Children 12-23 months had an over three-fold odds on stunting than children aged 0-5 months (OR 3.27; 95 \% 1.65-6.47). Children in the lowest wealth quintile had more than eight-fold odds on being severely stunted than children in the highest wealth quintile (AOR 8.48; 95 \% CI 3.19-22.57). Children whose mothers did not go to a health facility for ANC had over twice the odds on being severely stunted than children whose mothers did (AOR 2.58; 95 \% CI 1.19-5.58).

We repeated the analysis for children aged 0-35 months $(N=1937)$, excluding variables that were not available for children aged 24-35 months (see Additional file 2: Table S2). Child sex, child age and wealth quintile were significantly associated with severe stunting in the multivariate analysis. In addition, the odds on being stunted were significantly greater among children whose mothers did not complete primary education compared with those who completed senior high school (AOR 3.30, 95 \% CI 1.70-6.40).

\section{Discussion}

Our analysis examined the factors associated with stunting in children aged 0-23 months from three districts in Indonesia. The prevalence of stunting in this population (28.4 \%) was lower than the 2013 national survey data for Indonesia (32.9\%) [5] and similar to an 2004 survey in North Maluku Province in Indonesia (29.0 \%) [12].

We found that the odds on being stunted in households that drank untreated water was over three times higher if the household used an unimproved latrine, while in households that drank treated water the odds on stunting were $27 \%$ higher if the household used an unimproved latrine. Stunting was not associated with the household's source of drinking water or method used to dispose of a child's faeces. A significantly higher odds on stunting was observed among boys, older children and poorer households. For children aged 0-35 months, 
Table 4 Risk factors for severe stunting in children aged 0-23 months

\begin{tabular}{|c|c|c|c|c|c|c|c|c|c|}
\hline \multirow[t]{2}{*}{ Factors } & & \multirow{2}{*}{$\begin{array}{l}\text { Severely } \\
\text { stunted } \\
(\%)\end{array}$} & \multirow[t]{2}{*}{$N$} & \multicolumn{3}{|c|}{ Unadjusted (bivariate) } & \multicolumn{3}{|c|}{ Adjusted (multivariate) } \\
\hline & & & & $\mathrm{OR}$ & $(95 \% \mathrm{Cl})$ & $P$ & $\mathrm{OR}$ & $(95 \% \mathrm{Cl})$ & $P$ \\
\hline \multirow[t]{2}{*}{ Sex } & Boys & 8.1 & 689 & 1.61 & $(0.97-2.67)$ & 0.07 & 1.77 & $(1.07-2.93)$ & 0.03 \\
\hline & Girls & 5.3 & 677 & 1.00 & & & 1.00 & & \\
\hline \multirow[t]{3}{*}{ Age of child } & $12-23$ months & 8.8 & 716 & 3.34 & $(1.64-6.79)$ & 0.002 & 3.27 & $(1.65-6.47)$ & $<0.001$ \\
\hline & 6-11 months & 5.9 & 341 & 2.17 & $(0.91-5.15)$ & & 1.92 & $(0.82-4.48)$ & \\
\hline & $0-5$ months & 2.9 & 309 & 1.00 & & & 1.00 & & \\
\hline \multirow[t]{2}{*}{ Breastfeeding within one hour of birth } & No & 8.4 & 438 & 1.47 & $(0.94-2.29)$ & 0.09 & & & \\
\hline & Yes & 5.9 & 928 & 1.00 & & & & & \\
\hline \multirow[t]{2}{*}{ Age-appropriate feeding } & No & 8.3 & 812 & 1.52 & $(0.96-2.41)$ & 0.07 & & & \\
\hline & Yes & 4.5 & 554 & 1.00 & & & & & \\
\hline \multirow[t]{4}{*}{ Mother's age } & $\geq 40$ years & 9.8 & 92 & 0.87 & $(0.27-2.88)$ & 0.65 & & & \\
\hline & 30-39 years & 5.6 & 516 & 0.57 & $(0.21-1.52)$ & & & & \\
\hline & 20-29 years & 6.8 & 704 & 0.63 & $(0.25-1.59)$ & & & & \\
\hline & $<20$ years & 11.1 & 54 & 1.00 & & & & & \\
\hline \multirow[t]{4}{*}{ Mother's education } & No or incomplete primary & 19.4 & 196 & 6.81 & $(3.59-12.91)$ & $<0.001$ & & & \\
\hline & Completed primary & 8.3 & 229 & 2.57 & $(1.38-4.77)$ & & & & \\
\hline & Completed junior high & 5.1 & 333 & 1.77 & $(0.97-3.23)$ & & & & \\
\hline & Completed senior high & 3.0 & 608 & 1.00 & & & & & \\
\hline \multirow[t]{2}{*}{ Number of household members } & $>4$ & 7.7 & 816 & 1.39 & $(0.82-2.37)$ & 0.22 & & & \\
\hline & $\leq 4$ & 5.3 & 550 & 1.00 & & & & & \\
\hline \multirow[t]{5}{*}{ Wealth quintile } & Lowest & 17.0 & 294 & 8.62 & $(3.36-22.12)$ & $<0.001$ & 8.48 & $(3.19-22.57)$ & $<0.001$ \\
\hline & Second & 6.1 & 277 & 2.80 & $(1.08-7.28)$ & & 2.93 & $(1.12-7.70)$ & \\
\hline & Third & 3.3 & 273 & 1.49 & $(0.53-4.22)$ & & 1.66 & $(0.59-4.69)$ & \\
\hline & Fourth & 3.9 & 256 & 1.77 & $(0.62-5.08)$ & & 1.89 & $(0.65-5.45)$ & \\
\hline & Highest & 2.3 & 266 & 1.00 & & & 1.00 & & \\
\hline \multirow[t]{2}{*}{ Sanitation } & Unimproved & 10.5 & 532 & 2.20 & $(1.40-3.47)$ & 0.001 & & & \\
\hline & Improved & 4.3 & 834 & 1.00 & & & & & \\
\hline \multirow[t]{2}{*}{ Safe disposal of child's faeces } & Unsafe & 8.9 & 773 & 1.92 & $(1.19-3.12)$ & 0.008 & & & \\
\hline & Safe & 3.9 & 593 & 1.00 & & & & & \\
\hline \multirow[t]{2}{*}{ Use of soap for hand washing } & Not use soap & 10.0 & 611 & 2.04 & $(1.28-3.24)$ & 0.003 & & & \\
\hline & Use soap & 4.1 & 755 & 1.00 & & & & & \\
\hline \multirow[t]{2}{*}{ Water source } & Unimproved & 6.6 & 935 & 1.04 & $(0.57-1.93)$ & 0.89 & & & \\
\hline & Improved & 7.0 & 431 & 1.00 & & & & & \\
\hline \multirow[t]{2}{*}{ Water treatment } & Untreated & 14.7 & 136 & 2.19 & $(1.20-3.98)$ & 0.011 & & & \\
\hline & Treated & 5.9 & 1230 & 1.00 & & & & & \\
\hline \multirow{2}{*}{$\begin{array}{l}\text { Number of ANC visits of mother } \\
\text { during pregnancy }\end{array}$} & $<4$ & 12.1 & 91 & 1.35 & $(0.45-4.02)$ & 0.59 & & & \\
\hline & $\geq 4$ & 6.4 & 1275 & 1.00 & & & & & \\
\hline \multirow{2}{*}{$\begin{array}{l}\text { Doctor/midwife provided ANC to } \\
\text { mother during pregnancy }\end{array}$} & No & 21.9 & 64 & 3.23 & $(1.51-6.92)$ & 0.003 & & & \\
\hline & Yes & 6.0 & 1302 & 1.00 & & & & & \\
\hline \multirow[t]{2}{*}{ ANC in private or public health facility } & No & 25.0 & 48 & 4.04 & $(1.68-9.70)$ & 0.002 & 2.58 & $(1.19-5.58)$ & 0.02 \\
\hline & Yes & 6.1 & 1318 & 1.00 & & & & & \\
\hline \multirow{2}{*}{$\begin{array}{l}\text { Mother participates in decisions on } \\
\mathrm{HH} \text { food purchases }\end{array}$} & Yes & 6.8 & 1211 & 0.91 & $(0.47-1.76)$ & 0.77 & & & \\
\hline & No & 6.5 & 155 & 1.00 & & & & & \\
\hline
\end{tabular}


Table 4 Risk factors for severe stunting in children aged 0-23 months (Continued)

\begin{tabular}{lllllll}
\hline $\begin{array}{l}\text { Mother participates in decisions on } \\
\text { what food is cooked for HH }\end{array}$ & Yes & 7.3 & 1223 & 3.09 & $(1.13-8.44)$ & 0.028 \\
Mother participates in decisions on & Yes & 2.1 & 143 & 1.00 & \\
$\begin{array}{l}\text { Mood given to child } \\
\text { foos }\end{array}$ & No & 6.8 & 1300 & 1.11 & $(0.40-3.08)$ & 0.85 \\
Mother participates in decisions on & Yes & No & 6.1 & 66 & 1.00 & \\
seeking health care for child & No & 6.9 & 1180 & 1.22 & $(0.64-2.34)$ & 0.55 \\
\end{tabular}

the odds on stunting were significantly greater among children whose mothers had completed less years of schooling; otherwise the results were similar to children aged 0-23 months.

The relationship between WASH and nutritional status has not been thoroughly investigated in Indonesia. Recent analyses of cross-sectional survey data on the determinants of child stunting in Indonesia $[12,13]$ did not consider or comment on associations with WASH variables. A study examining community determinants of child growth in an Indonesian tea plantation found that the density of latrines was significantly associated with improved nutritional status of children aged 6-18 months [14] but the analysis did not differentiate between improved and unimproved latrines. An evaluation of a sanitation programme in East Java found reductions in the prevalence of soil-transmitted helminths and improvements in height, weight and weight-for-height among children living in households that had an unimproved latrine at baseline; the effects were significant only for non-poor households, which were more likely to build latrines as a result of the programme [15].

Elsewhere, evidence of the association between sanitation and stunting in low and middle-income countries is growing. Analysis of data collected in eight countries across three continents showed that incremental improvements in sanitation were significantly associated with increases in child height [16]. Using data from 172 Demographic and Health Surveys (DHS) between 1986 and 2007, Fink et al. [17] examined within-country variations in stunting and sanitation and showed that the odds on stunting were lower in households that had access to improved sanitary facilities (OR: 0.73, $95 \% \mathrm{CI}$ 0.71-0.75). More recently, Spears [18] used DHS data from 65 countries to examine inter-country variations in stunting and sanitation, and estimated that variations in stunting explain $54 \%$ of international variation in child height. In addition, a variety studies from individual countries involving cross-sectional survey analysis (India $[19,20])$, longitudinal studies (Peru [21]) and operational research (Ethiopia [22]) indicate that improved sanitation is important for the linear growth of children.

The global evidence on the association between water source and stunting is less clear. Analysis of DHS data from 70 low and middle income countries [17] showed that an improved water source was associated with a lower odds on stunting (OR: 0.92, 95 \% CI 0.89-0.94). Other studies have indicated that the protective effect of improved water is conditional on other WASH factors. Using data collected in the 1980s from eight countries in Africa, South Asia and South America, Esrey [16] found that the effect of water supply on child height was small and only positive among rural children when both improved sanitation was optimal and water services were available. More recently, Rah et al. [20] found that the protective effects of the mother or caregiver's reported personal hygiene practices against stunting in India were stronger when they were accompanied by household access to piped water. In our study, the interaction between household treatment of drinking water and sanitation suggests that household treatment of water may provide some protective effect in households that have unimproved sanitation.

A meta-analysis of data from 14 cluster-randomized trials conducted in 10 low and middle income countries found a small benefit of WASH interventions (specifically solar disinfection of water, provision of soap, and improvement of water quality) on the height of in children under five years of age [23]. The analysis was constrained by the lack of studies of high methodological quality, particularly for sanitation.

Poor WASH facilities and behaviours may impact on child nutritional status by causing diarrhoea [24], intestinal worm infections [25], or environmental enteropathy [26]. These infections and conditions directly affect nutritional status through variations pathways including loss of appetite, loss of host tissues, maldigestion or malabsorption of nutrients, chronic immune activation, and other responses to infection that divert the use of nutrients and energy, such as fever.

We conducted a similar analysis to identify the determinants of severe stunting in children aged 0-23 months and 0-35 months. The direction of the bivariate associations between the WASH variables and severe stunting were similar to total stunting, however the associations did not remain significant in the multivariate model, possibly because the prevalence of severe stunting was relatively low in this population 
(6.7 \% in children aged 0-23 months and $7.7 \%$ in children aged 0-35 months).

Our findings of a higher odds on stunting and severe stunting among boys, older children, lower household wealth, and lower maternal education are consistent with findings in Indonesia $[12,13]$ and other low and middleincome countries [27-30].

There are several limitations in our analysis. First, we used cross-sectional data and so the analysis cannot provide evidence of a causal relationship between stunting and determinants. Randomized-controlled trials on the relation between sanitation and health is difficult to generate due to complexities in behaviour change of communities, variations in technological requirements, and the multitude of faecal contamination pathways $[31,32]$. Second, data on personal and household practices was based on the mother's recall, which may have been subject to bias. Third, the analysis did not include data on the mother's nutritional status or the child's birth weight or length, which are known predictors of stunting [13, 33]; birth weight data was available for only 823 children, while measurements of mother's height and body mass index were not collected. However, we included indicators on household socio-economic status and health seeking behaviour that may partly explain mothers' nutrition and health status. Fourth, children with chronic diseases were excluded and there could have been a relationship between the presence of some chronic diseases and stunting. However, these diseases are rarely diagnosed in children aged less than two years in Indonesia and so very few children would have been excluded. Fifth, the analysis did not include all determinants of household water quality, including safe water handling and storage prior to drinking [34]. Finally, the data were obtained from three districts, which are not necessarily representative of the country as a whole but all had a high prevalence and/or burden of stunting and represent different typologies in Indonesia.

Despite these limitations, the findings from this study have exposed associations between stunting and both household sanitation and water treatment in Indonesia that have previously not been reported. The findings are of immense concern to Indonesia because $20 \%$ of all households in Indonesia, including $29 \%$ of households in rural areas, defecate in the open, which amounts to over 51 million people [10], the second highest number in the world. They support the hypothesis that the variations between provinces in the proportion of households with no access to an improved latrine (Fig. 1) may partly explain the variations in stunting prevalence across Indonesia.

With stagnating levels of stunting a threat to the human and economic prosperity in Indonesia, these findings should be used to argue for greater prioritisation, capacity and investment in WASH interventions as part of the government's efforts to reduce stunting in the first two years of life. Operational research is needed to generate further evidence on how to effectively converge and integrate WASH interventions with other stunting reduction interventions for greater impact on stunting prevalence and burden. The Sustainable Development Goals may present opportunities in this respect; increased collaboration between nutrition (Goal 2) and WASH (Goal 6) could be greatly encouraged by strong focus on high quality data and evidence of progress (Goal 17) [35, 36].

\section{Conclusions}

In conclusion, our analysis shows that household sanitation and treatment of drinking water were strong predictors of stunting in a population of children aged $0-23$ months in Indonesia. The findings add to the growing body of national and global evidence on the linkages between WASH and linear growth in early life, and indicate that policy and programme actions to address stunting should give greater attention to WASH interventions in Indonesia.

\section{Additional files}

Additional file 1: Table S1. Risk factors for stunting in children age 0-35 months $(N=1937)$. (DOCX $21 \mathrm{~kb})$

Additional file 2: Table S2. Risk factors for severe stunting in children age 0-35 months $(N=1937)$. (DOCX $21 \mathrm{~kb})$

\section{Abbreviations}

ANC, antenatal care; AOR, adjusted odds ratio; $\mathrm{Cl}$, confidence interval; DHS, Demographic and Health Survey; IYCF, infant and young child feeding; MYCNSIA, Maternal and Young Child Nutrition Security Initiative; OR, odds ratio; SUN, Scaling Up Nutrition; WASH, Water, sanitation and hygiene.

\section{Acknowledgments}

The MYCNSIA baseline survey was led by Siti Muslimatun, Southeast Asian Ministers of Education Organization, Regional Centre for Food and Nutrition, and Endang L. Achadi, University of Indonesia. We acknowledge the contribution of Nisaa' R Wulan and Raehan Mawardi to the data analysis and thank Christine Rudert and Chander Badloe for helpful feedback and suggestions on the manuscript. The opinions expressed in this paper are those of the authors and do not necessarily represent an official position by UNICEF.

\section{Funding}

The European Union provided funding for the data collection, data management, data analysis and manuscript writing via an award for the MYCNSIA project (DCI-FOOD/2010/258-665). The European Union was not involved in the design of the study, the collection, analysis, and interpretation of data, or in writing the manuscript.

\section{Availability of data and materials}

The dataset supporting the conclusions of this article will be made available upon requests that are in-line with UNICEF's procedures for ethical standards in research, evaluation, data collection and analysis (jakarta@unicef.org).

\section{Authors' contributions}

HT conceptualized the data analysis, interpreted the data and drafted and finalized the manuscript. AAC contributed to the design of the analysis, interpreted the data and contributed to the draft manuscript. SKS contributed to the design of the analysis, conducted the statistical analysis 
and contributed to the draft manuscript. RN contributed to the draft manuscript. All authors read and approved the final manuscript.

\section{Competing interests}

The authors declare that they have no competing interests.

\section{Consent for publication}

Not applicable.

\section{Ethics approval and consent to participate}

The survey received ethical clearance from the Ethical Committee of the Faculty of Medicine, Jakarta, University of Indonesia, reference number 550/ PT02.FK/ETIK/2011. Individual consent to participate in the survey was given by the child's primary caregiver.

\section{Author details}

${ }^{1}$ UNICEF Indonesia, World Trade Center 6 (10th Floor), Jalan Jenderal Sudirman Kav. 31, Jakarta 12920, Indonesia. ${ }^{2}$ Faculty of Public Health, University of Airlangga, Banyuwangi Campus, Jalan Wijaya Kusuma No 113, Kecamatan Giri, Banyuwangi, Indonesia.

Received: 10 November 2015 Accepted: 20 June 2016 Published online: 29 July 2016

\section{References}

1. International Food Policy Research Institute. Global nutrition report 2014 actions and accountability to accelerate the world's progress on nutrition. Washington DC: International Food Policy Research Institute; 2014.

2. Black RE, Victora CG, Walker SP, Bhutta ZA, Christian P, de Onis M et al Maternal and child undernutrition and overweight in low-income and middle-income countries. Lancet. 2013; doi:10.1016/S0140-6736(13)60937-x.

3. Horton S, Steckel RH. Malnutrition: Global economic losses attributable to malnutrition 1900-2000 and projections to 2050. In: Lomborg B, editor. How much have global problems cost the world? A scorecard from 1900 to 2050. Cambridge: Cambridge University Press; 2013.

4. UNICEF. Improving child nutrition: the achievable imperative for global progress. New York: UNICEF; 2013.

5. National Institute of Research and Development, Ministry of Health. Basic health research survey (Riset kesehatan dasar). Jakarta: National Institute of Research and Development, Ministry of Health; 2013.

6. Republic of Indonesia. Policy framework. National Movement on Nutritional Awareness Within the Framework of the First Thousands Days of Life. Jakarta: Republic of Indonesia; 2012.

7. National Development Planning Agency. Nutrition: Health Sector Review 2014. National Development Planning Agency: Jakarta; 2014.

8. Rabe-Hesketh S, Skrondal A. Multilevel and Longitudinal Modeling Using Stata, third edition. Volume II: Categorical Responses, Counts, and Survival. Texas: Stata Press; 2012. p. 974.

9. World Health Organization, Multi-Center Growth Study Group. WHO child growth standards based on length/height, weight and age. Acta Paediatr. 2006:450:76-85

10. World Health Organization. Indicators for assessing infant and young child feeding practices. Part 1. Geneva: World Health Organization; 2008.

11. World Health Organization, UNICEF. Progress on drinking water and sanitation: 2015 update. World Health Organization and UNICEF Joint Monitoring Programme. Geneva: World Health Organization and UNICEF; 2015.

12. Ramli, Agho KE, Inder KJ, Bowe SJ, Jacobs J, Dibley MJ. Prevalence and risk factors for stunting and severe stunting among under-fives in North Maluku province of Indonesia. BMC Pediatr. 2009:9:64.

13. Semba RD, de Pee S, Sun K, Sair M, Akhter N, Bloem MW. Effect of parental formal education on risk of child stunting in Indonesia and Bangladesh: a cross-sectional study. Lancet. 2008;371:322-8.

14. Paknawin-Mock J, Jarvis $L$, Jahari AB, Husaini MA, Pollitt E. Community-level determinants of child growth in an Indonesian tea plantation. Eur J Clin Nutr. 2000;54 Suppl 2:S28-42.

15. Cameron L, Shah M, Olivia S. Impact evaluation of a large-scale rural sanitation project in Indonesia, Water and Sanitation Program. Impact Evaluation Series No. 83, Policy Research Working Paper WPS6360. Washington: World Bank; 2013.

16. Esrey S. Water, waste and well-being: a multi-country study. Am J Epidemiol. 1996;143(6):608-23.
17. Fink G, Gunther I, Hill K. The effect of water and sanitation on child health: evidence from the demographic and health surveys 1986-2007. Int J Epidemiol. 2011;40:1196-204

18. Spears D. How much international variation is child height can sanitation explain? World Bank policy research paper, No WPS 6351. Washington DC: World Bank; 2013.

19. Spears D, Ghosh A, Cumming O. Open defecation and childhood stunting in India: an ecological analysis of new data from 112 districts. PLOS ONE. 2013;doi:10.1371/journal/pone.0073784.

20. Rah JH, Cronin AA, Badgaiyan B, Aguayo VM, Coates S, Ahmed S. Household sanitation and personal hygiene practices are associated with child stunting in rural India: a cross-sectional analysis of surveys. Brit Med J Open. 2015; doi:10.1136/bmjopen-2014-005180.

21. Checkley W, Gilman RH, Black RE, Epstein LD, Cabrera L, Sterling CR, et al. Effect of water and sanitation on childhood health in a poor Peruvian periurban community. Lancet. 2004;363:112-8.

22. Fenn B, Bulti AT, Nduna T, Duffield A, Watson F. An Evaluation of an operations research project to reduce childhood stunting in a food-insecure area in Ethiopia. Public Health Nutr. 2012;15:1746-54.

23. Dangour AD, Watson L, Cumming O, Boisson S, Che $Y$, Velleman $Y$ et al. Interventions to improve water quality and supply, sanitation and hygiene practices, and their effects on the nutritional status of children. Cochrane Database of Systematic Reviews, 2013;doi:10.1002/14651858.CD009382.pub2.

24. Briend $\mathrm{A}$. Is diarrhoea a major cause of malnutrition among the under-fives in developing countries? A review of available evidence. Eur J Clin Nutr. 1990:44(9):611-28

25. Hall A, Hewitt G, Tuffrey $V$, de Silva N. A review and meta-analysis of the impact of intestinal worms on child growth and nutrition. Maternal and Child Nutrition. 2008;4:118-236.

26. Humphrey $\mathrm{JH}$. Child undernutrition, tropical enteropathy, toilets, and handwashing. Lancet. 2009;374:1032-5.

27. Wamani H, Åstrøm AN, Peterson S, Tumwine JK, Tylleskär T. Boys are more stunted than girls in Sub-Saharan Africa: a meta-analysis of 16 demographic and health surveys. BMC Pediatrics. 2007;doi:10.1186/1471-2431-7-17.

28. Moestue $\mathrm{H}$, Huttly $\mathrm{S}$. Adult education and child nutrition: the role of family and community. J Epidemiol Commun H. 2008;62:153-9.

29. Prentice AM, Ward KA, Goldberg GR, Jarjou LM, Moore SE, Fulford AJ, et al. Critical windows for nutritional interventions against stunting. Am J Clin Nutr. 2013;doi: 10.3945/ajcn.112.052332.

30. Bredenkamp C, Buisman LR, de Poel EV. Persistent inequalities in child undernutrition: evidence from 80 countries, from 1990 to today. Int J Epidemiol. 2014; doi: 10.1093/ije/dyu075.

31. Luby SP. Is targeting access to sanitation enough? Lancet Global Health. 2014:doi:10.1016/S2214-109X(14)70326-2.

32. Schmidt WPT. The elusive effect of water and sanitation on the global burden of disease. Trop Med Int Health. 2014;doi:10.1111/tmi.12286.

33. Schmidt MK, Muslimatun S, West CE, Schultink W, Gross R, Hautvast JGAJ. Nutritional status and linear growth of Indonesian infants in West Java are determined more by prenatal environment than by postnatal factors. J Nutr. 2002;132:2202-7.

34. Sodha SV, Menon M, Trivedi K, Ati A, Figueroa ME, Ainslie R, Wannemuehler K, Quick R. Microbiologic effectiveness of boiling and safe water storage in South Sulawesi, Indonesia. J Water Health. 2011:9:577-85.

35. United Nations. Proposal for sustainable development goals, outcome of United Nations General Assembly Open Working Group on Sustainable Development Goals. New York: United Nations; 2014

36. Cronin AA, Badloe C, Torlesse H, Nandy RK. Water, Sanitation and Hygiene (WASH) - moving the policy agenda forward in the post 2015 Asia. Asia Pac Pol Studies. 2015:doi:10.1002/app5.90. 